\title{
How Metallic Fe Controls the Composition of its Native Oxide
}

\author{
Sebastien Couet, ${ }^{1, *}$ Kai Schlage, ${ }^{1}$ Karel Saksl, ${ }^{2}$ and Ralf Röhlsberger ${ }^{1}$ \\ ${ }^{1}$ Deutsches Elektronen Synchrotron (DESY), Notkestraße 85, 22603 Hamburg, Germany \\ ${ }^{2}$ Institute of Materials Research, Slovak Academy of Sciences, Watsonova 47, 04353 Kosice, Slovak Republic
}

(Received 18 April 2008; published 1 August 2008)

\begin{abstract}
We have studied in situ the oxidation of ultrathin iron layers and monitored the chemical changes induced by subsequent deposition of Fe metal using hard x-ray absorption spectroscopy. The site sensitivity of the technique allows us to quantify the composition of the layer throughout the oxidation or deposition process. It is found that the thin native oxide incorporates a significant fraction of Fe atoms remaining in a metallic configuration even in the saturated state. Subsequent deposition of Fe leads to a complete reduction of the oxide that adopts an $\mathrm{FeO}$-like structure containing $\mathrm{Fe}^{2+}$ sites only.
\end{abstract}

DOI: 10.1103/PhysRevLett.101.056101

In recent years, intensive research has been conducted on transition metal oxide compounds, thin films, and multilayers due to their potential use as building blocks in magnetic structures like spin valves and magnetic tunnel junctions (for a review, see [1] and references therein). Recently, metal-native-oxide multilayers (MNOM, produced by metal deposition and subsequent oxygen exposure) have been proposed as new compounds combining high magnetization and low conductivity [2]. In these systems, the buried oxide possesses magnetic properties that strongly deviate from any bulk iron oxide phase $[2,3]$. However, the influence of the surrounding metal on the structure of the oxide is not yet identified and prevents clear understanding of the observed magnetic properties of MNOM systems.

The oxidation of iron itself has been extensively studied in the past decades. Studies performed on polycrystalline [4] as well as on single crystalline [5] surfaces have shown that the oxidation of iron can be understood in the framework of the coupled current mechanism introduced by Fromhold and Cook more than 50 years ago [6]. While those experiments explain very well the observed growth kinetics, the actual composition of the native oxide is still a matter of debate and is obviously highly dependent on preparation parameters like temperature and surface orientation ([7-11] and references therein). X-ray absorption spectroscopy at the $L$ edges was recently used to characterize the extent of oxidation or reduction processes arising at the interface between $\mathrm{Fe}$ and other transition metal oxides ( $\mathrm{CoO}$ and $\mathrm{NiO}$ ) [12]. Remarkably, the interaction of $\mathrm{Fe}$ with its own oxide phases was never quantitatively studied before.

In this Letter, we present a quantitative $\mathrm{x}$-ray absorption spectroscopy (XAS) study of the oxidation of ultrathin iron layers and the changes induced by subsequent coverage with Fe. The sensitivity of the XAS signal to the oxidation state and the local environment of the absorbing atom is used to follow the evolution of the oxide's structure in a quantitative and site specific manner. The use of ultrathin layers (in contrast to other studies which used thick substrates $[4,5,7-9])$ allows one to directly resolve the intrin-
PACS numbers: 81.15.-z, 61.05.cj, 81.16.Pr

sic composition of the growing oxide and disentangle the influence of the substrate.

The experiment was performed in situ in a compact UHV deposition chamber (base pressure $1 \times 10^{-9} \mathrm{mbar}$ ) mounted on the A1 beam line of the DORIS III storage ring (DESY, Hamburg). The Fe $K$-edge absorption signal was recorded by measure of the total electron yield and fluorescence yield. Polycrystalline layers were grown by dc magnetron sputtering (Ar pressure $5 \times 10^{-3}$ mbar) on superpolished $\mathrm{Si}(001)$ substrates. Prior to the Fe deposition, a $6 \mathrm{~nm}$ thick Pd buffer layer was deposited to avoid unwanted oxidation at the $\mathrm{SiO}_{2} / \mathrm{Fe}$ interface. Iron layers, with thicknesses ranging from 0.4 to $2 \mathrm{~nm}$, were then deposited, stepwise oxidized to saturation (oxygen pressure $1 \times 10^{-5} \mathrm{mbar}$ ), and subsequently covered with a second Fe layer. Both the near edge (XANES) and the extended fine structure (EXAFS) spectra of the Fe $K$ edge were recorded ( $30 \mathrm{~min}$ acquisition time) at selected steps of the preparation. The influence of the residual gas pressure was checked by repeated XAS scans on a $0.8 \mathrm{~nm}$ thick metallic Fe layer, showing no detectable changes over several hours.

The different bulk iron oxide phases can be described as a combination of three different Fe sites distributed over interstitial positions in a close packed fcc oxygen lattice [13]. For quantitative XANES analysis, we used bulk iron oxide reference samples of $\mathrm{FeO}$ (single crystal, Mateck $\mathrm{GmbH}$, Germany), $\mathrm{Fe}_{3} \mathrm{O}_{4}$ and $\gamma-\mathrm{Fe}_{2} \mathrm{O}_{3}$ (both are powders diluted in a polyethylene matrix). As we do not expect that the oxidized layer adopts a long range crystalline ordering, the three bulk oxide references will rather be used to obtain the site specific concentration of $\mathrm{Fe}_{\text {oct }}^{2+}, \mathrm{Fe}_{\text {tet }}^{3+}$, and $\mathrm{Fe}_{\text {oct }}^{3+}$ in the layer. The subscripts "tet" and "oct" refer to ions in tetrahedral or octahedral positions.

Figure 1(b) shows the XANES spectra illustrating the stepwise oxidation of a $0.8 \mathrm{~nm}$ thick Fe layer. With increasing oxygen exposure, the onset of the absorption edge shifts to higher energies, indicating an increase of the overall oxidation state. Above $1300 \mathrm{~L}\left(1 \mathrm{~L}=10^{-6}\right.$ Torr s $)$, the saturation regime is reached and only little changes are still visible towards $6500 \mathrm{~L}$. To quantify the composition of the 

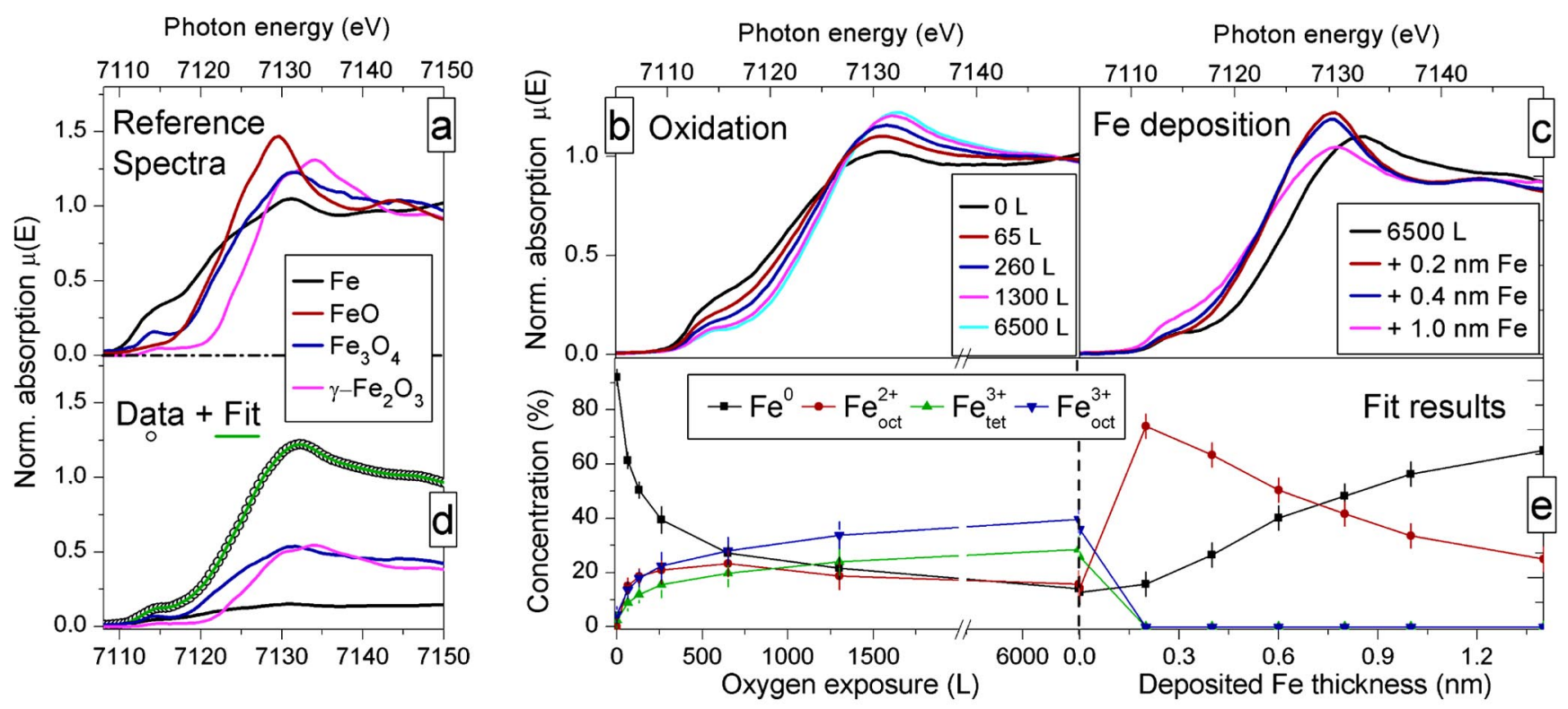

FIG. 1 (color online). (a) $\mathrm{Fe} K$-edge XANES spectra of reference samples for $\mathrm{Fe}, \mathrm{FeO}, \mathrm{Fe}_{3} \mathrm{O}_{4}$, and $\gamma$-Fe $\mathrm{Fe}_{2} \mathrm{O}_{3}$. (b) Evolution of the absorption edge position and shape for a $0.8 \mathrm{~nm}$ Fe layer exposed to an increasing amount of oxygen. (c) Evolution of the spectrum upon coverage of the saturated oxide by Fe. (d) Least square fitting of the saturated oxide spectra using a linear combination of reference spectra. (e) Evolution of the relative concentration of the different chemical sites as a function of oxygen exposure (left) and as a function of the Fe coverage on top of the saturated oxide (right).

layer, we fitted the spectrum of the oxidizing layer with a linear combination of the reference spectra. A fit of the saturated oxide, along with the contributions of the different references, is displayed in Fig. 1(d). Quantitative results are shown in the left-hand panel of Fig. 1(e). At low exposures, $\mathrm{Fe}_{\mathrm{oct}}^{2+}$ and $\mathrm{Fe}_{\text {oct }}^{3+}$ sites are created in equal quantities. Above $250 \mathrm{~L}$, the formation of $\mathrm{Fe}_{\text {oct }}^{2+}$ is hindered due to the reduced availability of $\mathrm{Fe}$ atoms at the surface while the number of $\mathrm{Fe}_{\text {oct }}^{3+}$ and $\mathrm{Fe}_{\text {tet }}^{3+}$ sites continues to grow. It is important to note that the oxide composition never corresponds to a stoichiometric bulk oxide. In principle, this could correspond to the formation of different stoichiometric oxides at crystallites exposing differently oriented surfaces to the oxygen. Grazing incidence x-ray diffraction measurements from a multilayered $\mathrm{Fe}$ /oxide sample indicated a lateral grain size of $10 \mathrm{~nm}$ for the $1.5 \mathrm{~nm}$ thick metal layers. No sign of crystalline structure was found for the oxide layers. We therefore expect that the oxidation does not lead in this case to stoichiometric oxide. The oxidation procedure was performed with Fe layer thicknesses ranging from 0.4 to $2 \mathrm{~nm}$. In each case, the oxidation curve follows a similar evolution as in Fig. 1(e). This indicates that the influence of an eventual nonuniformity of the initial $\mathrm{Fe}$ layers plays a minor role in the conclusions developed here.

Several studies on iron oxidation at room temperature (RT) reported the possible presence of metallic $\mathrm{Fe}$ in the oxide layer [2,5,9], without being able to disentangle the influence of the thick $\mathrm{Fe}$ substrate on the origin of the signal. The use of ultrathin Fe layers allows us to address this question in a more quantitative manner. In Fig. 1(e), we see that the concentration of Fe metal is not zero in saturation, although one would expect the layer to be completely oxidized, as reported in Ref. [2]. Figure 2 shows the evolution of the $\mathrm{Fe}^{0}$ concentration in the saturated $(6500 \mathrm{~L})$ oxide as a function of the initial Fe layer thickness. The $\mathrm{Fe}^{0}$ concentration remains around $12 \%$ (a constant fraction of the total $\mathrm{Fe}$ content) for all Fe thicknesses below $0.8 \mathrm{~nm}$, even for exposures as high as $25000 \mathrm{~L}$. Above $0.8 \mathrm{~nm}$, the proportion of metallic Fe increases almost linearly, indicating that a metallic layer remains below the oxide in the thicker layer. We can therefore expect that the metallic signal observed for the thin layers originates from $\mathrm{Fe}$ atoms which are still in a metallic environment and distributed throughout the oxide. While it is difficult to address the exact origin of this phenomenon, these measurements give new insight in the

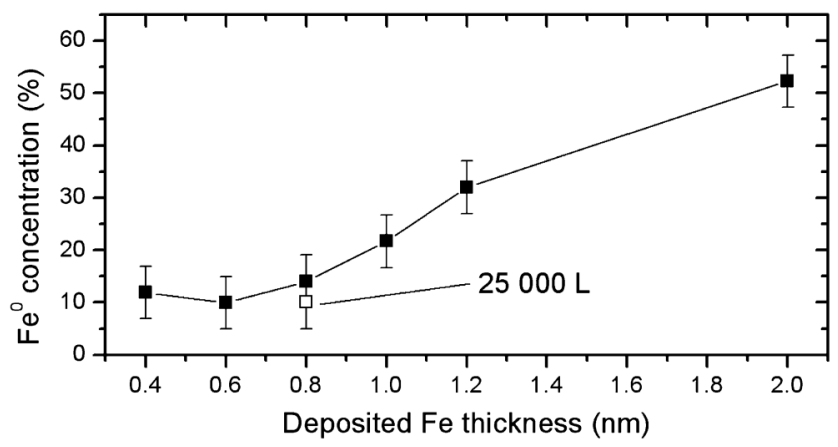

FIG. 2. Evolution of the $\mathrm{Fe}^{0}$ concentration in the saturated $(6500 \mathrm{~L})$ oxide as a function of the initial Fe layer thickness. 
previously reported possible presence of metallic $\mathrm{Fe}$ in an oxidized layer. These considerations should be taken into account when discussing the structure of such oxide.

As discussed by Beach et al. [2], buried native iron oxide layers (i.e., Fe oxidized at RT and covered with $\mathrm{Fe}$ ) possess structural and magnetic properties different from any bulk iron oxide phase, like a higher density and an anomalously high magnetic moment per atom. However, they were not able to relate the initial structure of the free oxide to the observed properties of the buried oxide. These questions can be directly addressed here by performing stepwise $\mathrm{Fe}$ deposition on top of the saturated oxide and analyzing the near edge structure of the $\mathrm{x}$-ray absorption signal. The corresponding XANES spectra are shown in Fig. 1(c). Starting with the saturated native oxide, a drastic change of the spectrum occurs upon coverage with only $0.2 \mathrm{~nm}$ of Fe. The profile obtained differs from what was observed during the oxidation and indicates a fundamental change of the Fe's chemical environment. The result of the fitting procedure is shown in the right-hand panel of Fig. 1(e). The change is attributed to a reduction of the native oxide to an $\mathrm{FeO}-$ like structure where only $\mathrm{Fe}_{\text {oct }}^{2+}$ sites are present. This was observed for every initial $\mathrm{Fe}$ layer thicknesses. In contrast to the free native oxide which exhibits a mixture

\section{Electron potential}
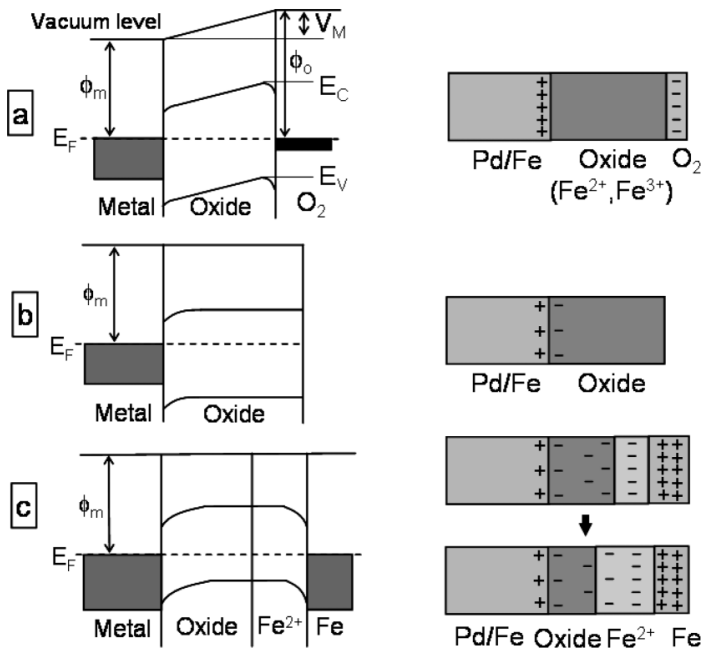

FIG. 3. Electron energy diagram (left-hand panels) and net charge distribution (right-hand panels) at different stages of the oxidation or reduction. $E_{F}$ is the Fermi level. $\phi_{m}$ and $\phi_{o}$ are the metal and $\mathrm{O}^{-}$level work functions, respectively. $E_{C}$ and $E_{V}$ are the energy levels of the conduction and valence band of the oxide, respectively. (a) Oxidation. The Mott potential $V_{M}=$ $\phi_{o}-\phi_{m}$ appears across the oxide layer, promoting the cation diffusion. (b) When the oxygen supply is shut off, the system is in equilibrium and only a small residual electric field is present at the metal-oxide interface due to the induced space charge in the oxide. (c) After deposition of Fe. The space charge in the oxide induces a net charge on the ultrathin Fe adsorbate which promote the $\mathrm{Fe}$ diffusion in the oxide. The right-hand inset shows the progressive growth of the $\mathrm{Fe}^{2+}$ layer. of several different chemical environments the buried native oxide adopts a well-defined composition made of $\mathrm{Fe}_{\text {oct }}^{2+}$ sites only (plus the original $\mathrm{Fe}^{0}$ content).

The fact that the $1.5 \mathrm{~nm}$ thick oxide layer is reduced to an $\mathrm{FeO}-$ like environment is remarkable. It implies that the Fe deposited on top of the layer can effectively diffuse to distances larger than $1 \mathrm{~nm}$ from the interface. At room temperature, thermal diffusion of cations is negligible and additional driving mechanisms are therefore expected to induce the diffusion. During the oxidation, the difference in the work functions of the metal and the oxygen $\mathrm{O}^{-}$level leads to the appearance of a potential $V_{M}=\phi_{o}-\phi_{m}$ (the Mott potential [6]) across the oxide that induces a sufficiently large electric field to promote the cation diffusion through the oxide, as shown in Fig. 3(a). When the oxygen supply is shut off [Fig. 3(b)], $V_{M}$ disappears and only a residual electric field is present at the metal-oxide interface due to the space charges built up in the oxide [14]. Deposition of $\mathrm{Fe}$ on top of the saturated oxide results in a reduction of the first $\mathrm{Fe}^{3+}$ monolayer to $\mathrm{Fe}^{2+}$ (this process does not require cations transport). In the case of an ultrathin metal adsorbate like here, the space charges appearing at the new oxide-metal interface leads to a net charge on the metal overlayer. A strong electric field is created at the interface which promotes the diffusion of ions. It should be noted that the process of Fe-oxide reduction is not an artifact of the stepwise deposition of Fe described above but occurs also for deposition of thicker Fe layers in one step.

From the chemical point of view, only one type of coordination site exists in the buried oxide. However, the

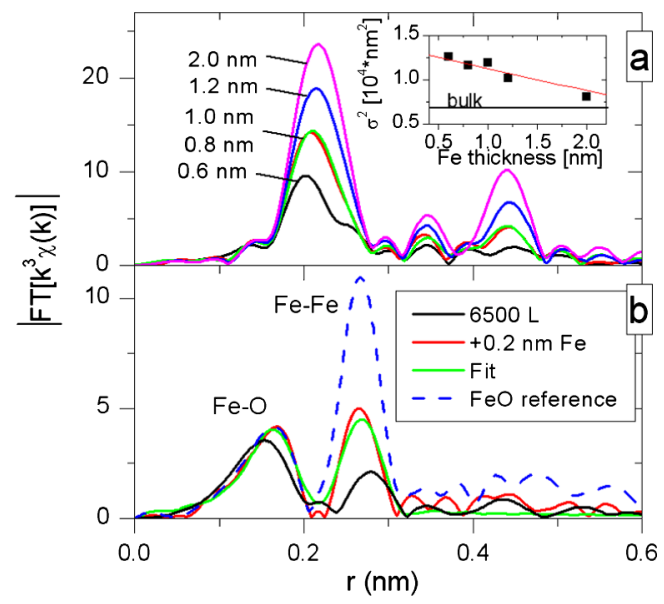

FIG. 4 (color online). (a) Fourier transform (FT) modules in the $r$ space of the EXAFS signal of unoxidized Fe layers of different thickness. The inset shows the evolution of the mean square displacement $\sigma^{2}$ of the first coordination shell. (b) FT of the EXAFS signal for the saturated native oxide $(6500 \mathrm{~L})$ and subsequent coverage with $0.2 \mathrm{~nm} \mathrm{Fe}(+0.2 \mathrm{~nm} \mathrm{Fe})$. The fitted radial distribution of the latter was obtained by fitting the filtered EXAFS signal constrained to the first two subshells $(r<$ $0.3 \mathrm{~nm}$ ). For comparison, a $\mathrm{FeO}$ reference for bulk $\mathrm{FeO}$ is given (dashed line). 
oxidation and reduction processes involve diffusion of cations through the oxide, which implies a modification of the structural quality of the lattice. To investigate the structural changes that take place during the oxidation process, we used the EXAFS part of the absorption signal, which is sensitive to the local order (i.e., nearest neighbor distance, coordination numbers) of the absorbing atoms. Figure 4(a) shows the Fourier transform of the EXAFS signal for different thicknesses of the initial, nonoxidized Fe layer. The intensity of the first coordination shell peak gradually increases with increasing layer thickness. Values of the mean square displacement $\sigma^{2}$ shown in the inset were extracted from the EXAFS fit procedure by fixing the total number of atoms $N$ in the first shell to 14 (bulk value for bcc Fe). While the values found for the thinnest layers are typical for highly disordered systems, $\sigma^{2}$ gradually decreases and almost reaches the bulk value of polycrystalline Fe for the $2 \mathrm{~nm}$ thick layer. The linear dependence of $\sigma^{2}$ on the initial amount of Fe deposited documents a smooth transition from ultrathin to thin films. This supports our interpretation that even the $0.4 \mathrm{~nm}$ thick layer behaves essentially as a continuous Fe layer. Figure 4(b) shows the Fourier transform modules of the EXAFS signal for the saturated oxide, for the reduced layer, and for the pure $\mathrm{FeO}$ reference sample. The $\mathrm{Fe}-\mathrm{O}$ (first nearest neighbor) peak for the reduced oxide layer matches the profile of the $\mathrm{FeO}$ reference, indicating that each $\mathrm{Fe}$ atom has the same direct environment as $\mathrm{FeO}$. However, the lower intensity of the $\mathrm{Fe}-\mathrm{Fe}$ peak compared to $\mathrm{FeO}$ points to a rather disordered structure. In fact, the results indicate a large $\sigma^{2}$ of $0.0165 \AA^{2}$. This value is 2 times larger than the value related to thermal vibration observed in $\mathrm{Fe}_{3} \mathrm{O}_{4}$ at room temperature [15], indicating a high degree of static disorder. In that sense, we see that deposition of Fe on top of a free native oxide leads to the formation of a layer with a well-defined local order that is comparable to that of bulk $\mathrm{FeO}$, but with a larger degree of structural disorder.

In summary, we have quantitatively studied the chemical change occurring during the oxidation of $\mathrm{Fe}$ layers as well as during the deposition of $\mathrm{Fe}$ on such oxide. The use of ultrathin layers combined with the site specificity of $\mathrm{x}$-ray absorption allowed us to quantitatively resolve the composition of the oxide at all stages of the oxidation or reduction process. We evidenced the presence of metallic $\mathrm{Fe}$ as a constituent of ultrathin iron layers that were oxidized at room temperature. Deposition of Fe on top of the multicomponent oxide leads to a reduction of this layer resulting in a single type of $\mathrm{Fe}_{\text {oct }}^{2+}$ sites arranged in an $\mathrm{FeO}$-like structure. These findings bring new insight into the previously reported properties of buried native iron oxides. The higher density and the higher magnetic moment per atom that was reported by Beach et al. [2] can be partly accounted for by the presence of metallic $\mathrm{Fe}$ in the layer. The fact that the native oxide covered with Fe exhibits a unique FeO-like structure raises new questions regarding mag- netic ordering. In particular, the RT magnetic order observed recently $[2,3]$ implies that the ordering temperature of the buried native oxide is much increased compared to the bulk Néel temperature of $\mathrm{FeO}\left(T_{N}=198 \mathrm{~K}\right)$. Understanding how the complex magnetic structure of such ultrathin oxide layers influence the electronic transport in $\mathrm{Fe} / \mathrm{FeO}$ multilayers might open interesting possibilities for spin electronic applications.

The results of this study are expected to be valid not only for iron, but apply to any metal with several coexisting oxidation states. This means that the coverage of a native metal oxide with the pure metal reduces the oxide to its lowest oxidation state and stabilizes it. The deposition procedure described here thus represents a simple way to obtain metal-metal-oxide heterostructures with unique electronic and magnetic properties.

The authors gratefully acknowledge E. Welter and D. Zajac for their help during the measurements at HASYLAB as well as Th. Diederich for his support. K. Saksl is indebted to the Slovak Grant Agency for Science for financial support (Grant No. 2/7196/27).

*Author to whom correspondence should be addressed. sebastien.couet@desy.de

[1] M. Finazzi, L. Duó, and F. Ciccacci, Surf. Sci. Rep. 62, 337 (2007).

[2] G. S. D. Beach, F. T. Parker, D. J. Smith, P. A. Crozier, and A. E. Berkowitz, Phys. Rev. Lett. 91, 267201 (2003).

[3] Th. Diederich, S. Couet, and R. Röhlsberger, Phys. Rev. B 76, 054401 (2007).

[4] P. C. J. Graat, M. A. J. Somers, A. M. Vredenberg, and E. J. Mittemeijer, J. Appl. Phys. 82, 1416 (1997).

[5] S. J. Roosendaal, A.M. Vredenberg, and F.H.P.M. Habraken, Phys. Rev. Lett. 84, 3366 (2000).

[6] A. T. Fromhold and E. L. Cook, Phys. Rev. 163, 650 (1967).

[7] H.-J. Kim, J.-H. Park, and E. Vescovo, Phys. Rev. B 61, 15284 (2000).

[8] K. Mori, M. Yamazaki, T. Hiraki, H. Matsuyama, and K. Koike, Phys. Rev. B 72, 014418 (2005).

[9] S. J. Roosendaal, B. van Asselen, J. W. Elsenaar, A. M. Vredenberg, and F. H. P. M. Habraken, Surf. Sci. 442, 329 (1999).

[10] M. W. Ruckman, J. Chen, M. Strongin, and E. Horache, Phys. Rev. B 45, 14273 (1992).

[11] G. Bhargava, I. Gouzman, C. M. Chun, T. A. Ramanarayanan, and S.L. Bernasek, Appl. Surf. Sci. 253, 4322 (2007).

[12] T. J. Regan, H. Ohldag, C. Stamm, F. Nolting, J. Lüning, J. Stöhr, and R. L. White, Phys. Rev. B 64, 214422 (2001).

[13] D. V. Dimitrov, K. Unruh, G.C. Hadjipanayis, V. Papaefthymiou, and A. Simopoulos, Phys. Rev. B 59, 14499 (1999).

[14] Q. Fu and T. Wagner, Surf. Sci. Rep. 62, 431 (2007).

[15] B. A. Wechsler, D. H. Lindsley, and C. T. Prewitt, Am. Mineral. 69, 754 (1984). 